\title{
Assistantes médicales: rapport annuel sur les activités de la FMH
}

\author{
Aisha Ahmed \\ Présidente des délégués cantonaux aux questions des assistantes médicales
}

Le Bureau des assistantes médicales a connu plusieurs changements importants. Le Dr Adrian Sury a démissionné pour raison d'âge de sa fonction de président des délégués cantonaux aux questions des assistantes médicales. Au secrétariat, Elisabeth Tröhler a elle aussi pu prendre une retraite bien méritée, après les nombreuses années où elle s'est occupée avec dévouement des questions concernant les assistantes médicales. Fort heureusement, nous avons pu recruter en la personne d'Annik Rüedi une successeure très compétente, qui est parvenue, malgré le peu de temps disponible, à se mettre rapidement au courant des affaires. Mme

\section{Le Bureau des assistantes médicales a connu plusieurs changements importants.}

Chiwith Baumberger travaille également au secrétariat depuis février 2020, comme spécialiste pour les assistantes médicales. Quant à moi, j’ai repris la présidence en janvier 2020. Peu après, nous avons tous été touchés par les conséquences de la pandémie de Covid-19, ce qui a bouleversé notre travail. Renonçant aux séances présentielles, nous nous sommes rabattus sur les plateformes en ligne. La formation des apprenties assistantes médicales a été fortement impactée, y compris les examens finaux. Chacun a donc dû faire preuve d'une grande flexibilité.

La répartition des tâches liées à la formation des assistantes médicales et des coordinatrices en médecine ambulatoire est actuellement la suivante:

- Délégués des sociétés cantonales de médecine aux questions des assistantes médicales: Dre A. Ahmed, présidente; vice-présidence: vacante

- Commission suisse pour le développement de la profession et la qualité: Dr J. Orellano, président

- Commission d'examen pour la procédure de qualification des assistantes médicales: Dr G. Printzen, président

- OrTra Formation professionnelle des assistantes médicales (Odamed): Mme M. Schenk (SVA), présidente; Dr G. Printzen (FMH), vice-président

- Commission de surveillance des cours interentre- prises: Dr C. Quinto, président; Dr J. Orellano, viceprésident

- Les demandes adressées au Comité central de la FMH par les organes concernés sont présentées par le Dr Carlos Quinto, membre du Comité central de la FMH et responsable du département Santé publique et professions de la santé.

- Secrétariat des assistantes médicales à la FMH: Mme Annik Rüedi et Mme Chiwith Baumberger

Le Bureau des assistantes médicales, composé des responsables susmentionnés, se réunit en règle générale une fois par année ou lorsque cela est nécessaire, pour discuter des problèmes ou projets éventuels.

La première année de formation selon l'ordonnance sur la formation professionnelle initiale révisée et le plan de formation MPA s'est déroulée sans difficultés majeures.

Les comptes rendus sur les activités menées dans les commissions susmentionnées sont rédigés par les responsables respectifs.

\section{Commission pour le développement de la profession et la qualité (Dr José Orellano)}

La pandémie de Covid-19 a paralysé dans une certaine mesure les activités de la Commission pour le développement de la profession et la qualité durant la période écoulée.

Les restrictions imposées par l'OFSP dans la formation avec les adaptations nécessaires pour la procédure de qualification ont constitué un véritable défi pour la commission. En effet, les modifications ont dû être élaborées et mises à disposition à très brève échéance. Trois cantons (TI, ZH, VD) n'ont hélas pas réalisé d'examens pratiques selon la décision du SEFRI. Nous ne sommes donc pas en mesure d'établir des comparaisons pour toute la Suisse.

En conséquence, la qualité des examens de fin d'apprentissage n'est pas congruente. Conformément aux instructions, les notes de l'examen de la branche «bases médicales», déjà réalisé en 2019, n'ont pas été retenues dans le certificat de fin d'apprentissage. Pour les 


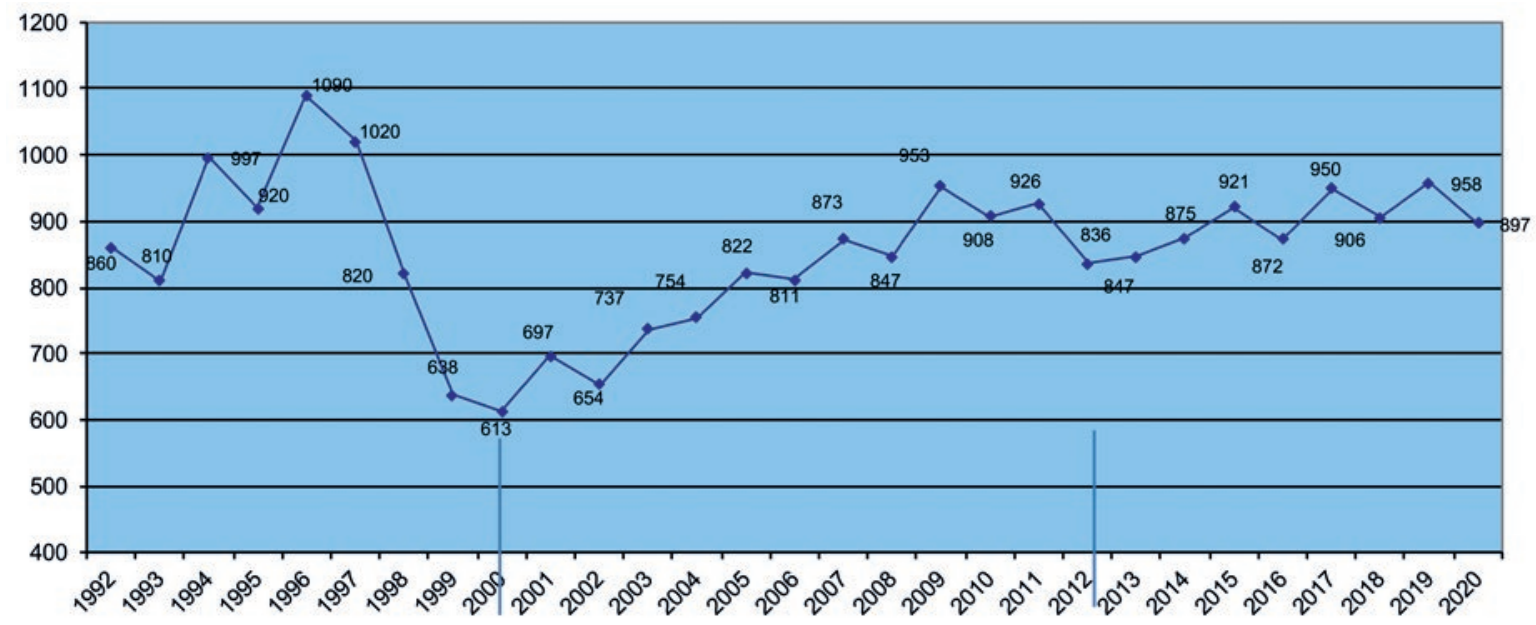

Formation selon l'ancien droit

Diplôme de fin d'apprentissage

Nouvelle ordonnance sur la formation

Statistique longitudinale des apprenties.

candidates en rattrapage, la Commission d'examen a réalisé un entretien professionnel nouvellement développé. Même avec ces modifications, les diplômes de fin d'apprentissage (CFC) 2020 sont équivalents à ceux des années précédentes.

\section{La pandémie de Covid-19 a paralysé dans une} certaine mesure les activités durant la période écoulée.

L'examen anticipé «Bases médicales» pour les diplômes 2021 a été annulé. La procédure de qualification se fondera sur les notes semestrielles obtenues dans les branches de base à la fin de l'apprentissage.

Compte tenu de cette contrainte supplémentaire, les autres déroulements en rapport avec les dispositions de la nouvelle ordonnance sur la formation professionnelle n'ont pas pu être élaborés correctement. Les travaux relatifs aux dispositions d'application ne sont pas encore terminés.

Je remercie l'ARAM et la SVA pour la bonne collaboration.

\section{Commission d'examen pour la procédure de qualification des assistantes médicales (Dr Gert Printzen)}

La Commission d'examen pour la procédure de qualification des assistantes médicales 2020 s'est consacrée activement à l'élaboration des documents d'examen. La conception d'un examen uniforme au niveau suisse représente régulièrement un véritable défi pour les groupes de spécialistes et leurs responsables, notamment pour satisfaire aux exigences d'un examen à la fois qualitativement exigeant et réalisable, mais aussi pour tenir compte des différences de pratique non négligeables entre la Suisse ro- mande, le Tessin et la Suisse alémanique suivant la discipline.

La procédure de qualification 2020 entrera dans les annales compte tenu de la pandémie de coronavirus (Covid-19). Dans ce contexte un peu particulier, 871 candidates se sont présentées aux examens, sous respect des règles d'hygiène et d'autres prescriptions. Le nombre de candidates a donc clairement baissé par rapport à l'année précédente (871 contre 1057). L’aperçu général des notes montre que le nombre de personnes ayant obtenu un résultat insuffisant a également fortement baissé ( $25=2,9 \%$ contre $99=9,4 \%$ ), alors que l'examen de cette année était plutôt difficile. La compilation des résultats d'examen s'est une fois de plus avérée fastidieuse, vu que les données des cantons n'étaient pas toutes disponibles au même moment.

La formation s'est déroulée selon la nouvelle ordonnance sur la formation professionnelle initiale. Par ailleurs, les travaux pour la mise en place d'un examen (écrit) en ligne en prévision des examens finaux selon la nouvelle ordonnance sur la formation professionnelle initiale, en 2022, se sont poursuivis. Cette manière de procéder permettra d'établir des examens numériques de haute qualité intégrés dans un pool et ensuite de créer des examens individuels à partir de ce

871 candidates se sont présentées aux examens, sous respect des règles d'hygiène et d'autres prescriptions.

dernier. Quant à savoir si ces examens seront réalisés en ligne ou par écrit, la question demeure indépendante du processus d'établissement de ceux-ci.

A l'instar des années précédentes, les tâches relatives aux examens ont également été considérables en 2020. C'est uniquement grâce à l'investissement important de toutes les personnes impliquées qu'il a été possible 
de les mener à bien. Je tiens à remercier chaleureusement tous les participants de leur engagement hors du commun et particulièrement constructif.

\section{Formation des assistantes médicales}

En 2020, 871 candidates se sont présentées aux examens d'assistante médicale. Parmi elles, 25 candidates $(2,9 \%)$ n'ont hélas pas réussi les examens.

\section{www.am-suisse.ch - www.fmh.ch}

Après la refonte du site internet de la FMH, toutes les informations concernant les assistantes médicales ont été transférées sur le site am-suisse.ch, où les employeurs trouvent tous les renseignements pertinents en la matière. Le site internet est continuellement mis à jour par le secrétariat des assistantes médicales de la FMH. La version allemande est accessible sur www.mpaschweiz.ch. Le site internet am-suisse.ch sera remanié en été 2021.

Aisha Ahmed

Présidente des délégués cantonaux aux questions des assistantes médicales Nussbaumstrasse 29 CH-3000 Berne 15

\section{Office de conciliation}

Durant la période sous revue, aucune nouvelle demande de conciliation n’a été déposée auprès
d'A.Kummer, avocat et notaire, chargé des conciliations pour la Suisse alémanique, et aucune affaire n'est en cours.

\section{En conclusion}

M. Bruno Gutknecht prendra une retraite bien méritée à la fin de l'année. Sa fonction au secrétariat central de la SVA sera reprise dès janvier 2021 par M. Peter Burkhalter. Nous remercions Bruno Gutknecht pour son engagement de longue date dans l'intérêt des assistantes médicales et lui souhaitons le meilleur pour la suite. Initialement, nous avions cette année aussi prévu de visiter les SwissSkills à Berne après l'assemblée annuelle des délégués aux questions des assistantes médicales. Comme cette manifestation s'est déroulée dans un cadre très restreint en raison du Covid-19, nous avons décidé de tenir notre assemblée annuelle une nouvelle fois à Lucerne.

Je remercie les secrétariats des assistantes médicales, les membres des différentes commissions, les associations professionnelles et toutes les autres personnes impliquées pour le travail constructif réalisé cette année, malgré les conditions difficiles.

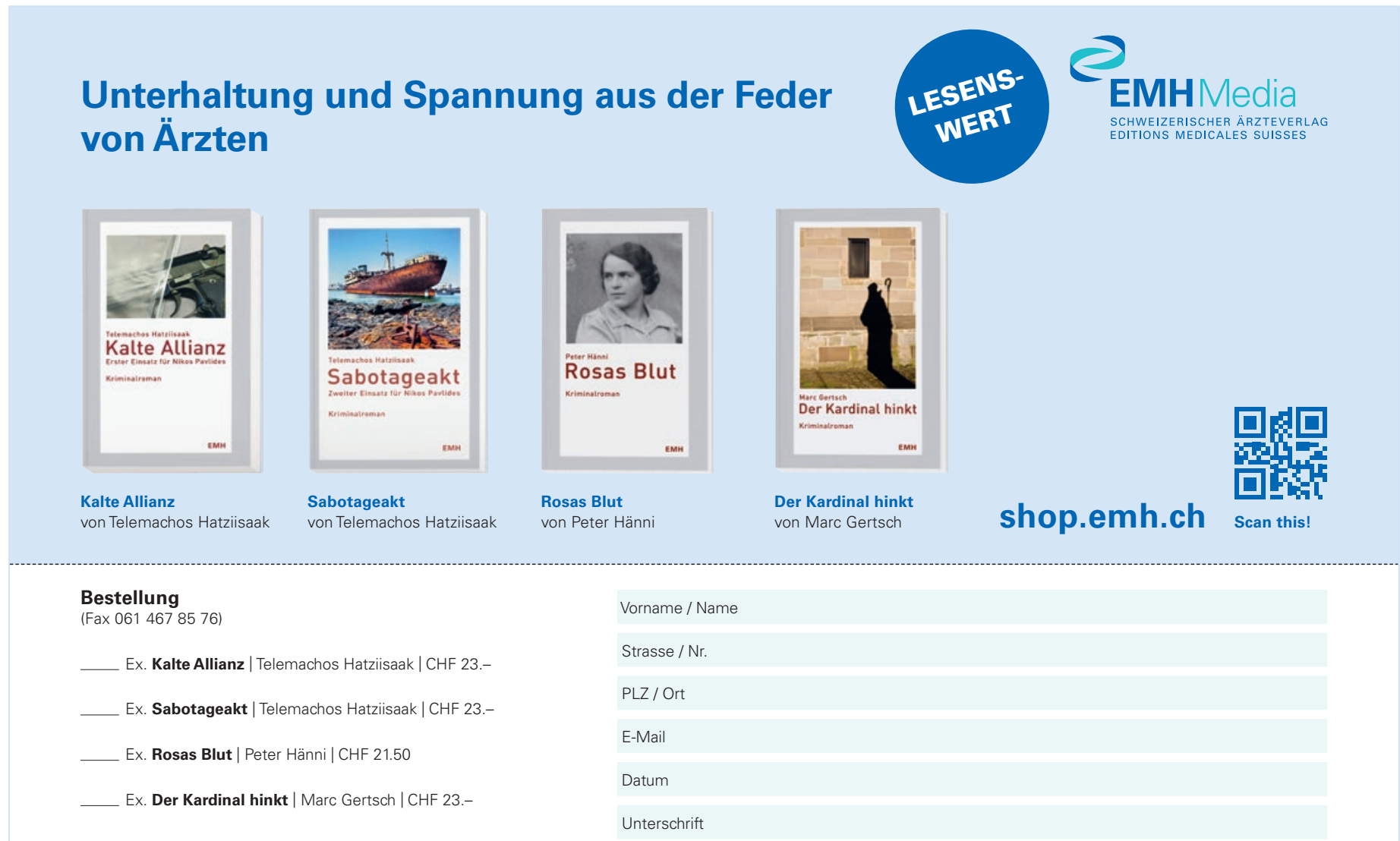

EMH Schweizerischer Ärzteverlag AG | Farnsburgerstrasse 8 | 4132 Muttenz auslieferung@emh.ch | shop.emh.ch 